\title{
Von Grenzgängern und neuen Heiligen. Imaginierte Nation zwischen Mexiko und den USA ${ }^{1}$
}

\author{
Inge Baxmann
}

\begin{abstract}
Migrationsbewegungen galten stets als Gefahr für nationalstaatliche Ordnungsmodelle, so auch an der Grenze zwischen Mexiko und den USA. Die Border-Crosser entwickelten transnationale Symbole und Praktiken der Zugehörigkeit, die den Nationalstaat in Frage stellten. Im Gegensatz dazu wurden sie von der Border-Culture, die seit den 1980er Jahren an der Grenze zwischen Mexiko und den USA entstanden war, als Vorreiter einer postnationalen Identität gefeiert. Die Border-Culture verstand sich als Gegenentwurf zur „Mexicanidad“, jenes imaginierten Nationenkonstruktes, das seit der mexikanischen Revolution und bis weit in die 1940er Jahre das Selbstverständnis des Landes bestimmte. Unabhängig von diesen Vorgaben entstanden religiöse Symboliken und Rituale jenseits der etablierten Kirchen sowie neue Formen eines nationalen Imaginären, das transnationale Formationen integrierte. Statt der Abschaffung der Nation trugen diese zur Flexibilisierung und Ausweitung der nationalen Ordnungen bei.
\end{abstract}

Keywords: Postnation; Migration; Border-Crosser

Widersprüche und Spannungen zwischen nationalstaatlichen Ordnungsmodellen und transnationalen Formationen sind ein Phänomen der Globalisierung. Kulturelle Mechanismen, über die moderne Nationalstaaten Zusammengehörigkeit symbolisierten, waren beispielsweise eine standardisierte Nationalsprache oder die Erfindung nationaler Traditionen durch die Folklorisierung des Volkes. Man suchte die ,imaginierte Gemeinschaft“2 der Nation zu untermauern und im Tiefenraum der Gesellschaft zu verankern. Den Nationen liegen solche Gemeinschaften zugrunde, während die politisch und juridisch verfassten Staaten die Kontrolle über dieses fest umrissene Territorium und seine Bevölkerung ausüben. Dass beide überhaupt zusammengehören sollten, war selbst Ausdruck eines nationalen Imaginären, das heute zunehmend in die Krise gerät. Denn sowohl der „Melting Pot“ in den USA wie die „Mexicanidad“ als Modelle der Nation beruhten auf der Vorstellung einer - wenn auch verschieden konzipierten - Einheit von Nation, Kultur und geographischem Raum. Grenzüberschreitung, Mischung der Sprachen, Kulturen und Räume, wie sie für Migranten an der Tagesordnung sind, waren nicht vorgesehen. Die Grenze zwischen Mexiko und den USA erscheint aus dieser Perspektive zugleich als ein Modellfall für die Produktion hegemonialer Macht wie für die Emergenz transnationaler

1 Der vorliegende Beitrag nimmt einige Aspekte aus meinem soeben erschienenen Buch auf (Baxmann 2007).

2 Zum Konzept der Nation als ,,imaginierter Gemeinschaft“ vgl. Anderson 2006 [1982]. 
Symbole und Praktiken der Zugehörigkeit. Sie wurde von beiden Staaten stets als notwendige Sicherung für die nationale Ordnung betrachtet; umgekehrt galten die Migrationsbewegungen von der einen zur anderen Seite als Risiko für eben diese Ordnung.

In der Geschichte Mexikos und der „Chicanos“ in den USA spielt die Grenze eine besondere Rolle: Sie geht auf den Krieg zwischen Mexiko und den USA zurück, der 1847 mit dem Sieg der USA endete. Mit dem Vertrag von Guadalupe Hidalgo erhielten die USA 1848 fast die Hälfte des mexikanischen Territoriums. Mexiko akzeptierte den Rio Grande als Grenze mit Texas und überließ den USA ihren Südwesten, der die heutigen Staaten von Arizona, Kalifornien, Nuevo Mexico, Utah, Nevada und Teile von Colorado umfasst. Damit wurden die Mexikaner gewissermaßen zu grenzüberschreitenden (und „grenzüberschrittenen“) Fremden in ihrem eigenen Land. Ihre Bezeichnung als „Chicanos“ bezeichnet diesen Zwischenraum. Ständige Migrationen brachten bald eine „Kultur“ zwischen drei Kulturen hervor: der mexikanischen, der angloamerikanischen und der eigenen Chicano-Kultur.

Die Diskrepanz zwischen globaler ökonomischer Integration und dem Fehlen von Modellen politischer Gemeinschaft für diese transnationalen Formationen ließ - zunächst spontan und dann seit den 1980er Jahren mit der Border-Culture bewusst - neue Modelle kultureller Zugehörigkeit entstehen, die sich als Gegenkonzepte zu nationalstaatlichen Formen der Gemeinschaftsbildung verstanden. Doch schon seit dem frühen 20. Jahrhundert verursachten transnationale Formationen, die mit der Entstehung neuer Symbolsysteme verbunden waren, eine Krise nationalstaatlicher Autorität, wie sie im Modell der „Mexicanidad“ eigentlich vorgesehen war.

\section{„Mexicanidad“: Symbolische Integration der Eliten für das Volk}

Das Projekt der „Mexicanidad“, wie es von der Politik des Kultur-und Bildungsministers José Vasconcelos entwickelt und vorangetrieben wurde, sollte als neue kulturelle Selbstbeschreibung die Leerstelle füllen, die nach der Revolution entstanden war. Es entsprach dem Wunsch nach Beendigung der Revolution und ihre Überführung in eine stabile soziale Ordnung. Die kulturelle Elite, die mit der Regierung Obregón (und später Lázaro Cárdenas) nach der mexikanischen Revolution von 1910 die Politik bestimmte, hatte in Europa, zumeist in Frankreich studiert und war mit den europäischen Wissenschaften und Künsten, insbesondere der Avantgarde, durchaus vertraut. Das Ziel dieser Intellektuellen war die Herstellung eines modernen Mexiko durch eine „Mexikanisierung“ der europäischen Moderne. Die neuesten europäischen Kunstrichtungen schienen gerade zur rechten Zeit zu kommen, um ins Mexikanische transponiert zu werden. Sie mussten nur populär gemacht werden. Gegen die Bedrohung durch die USA, gegen die regionale und ethnische Zersplitterung des Landes, aber auch aus Furcht vor den Folgen der Migration zwischen beiden Ländern setzte man auf die Entwicklung einer gemeinschaftsstiftenden volkstümlichen Nationalkultur. Nach der Revolution waren die Akteure mit einer komplexen Situation konfrontiert. Mexiko bestand aus einem Flickenteppich von sozialen Klassen und Regionen (Hodges/Gandy 2002, 27/28). Für die postrevolutionäre Politik bedeutete dies vor allem, drei grundlegend verschiedene ethnische Gruppen zusammenbringen zu müssen: weiße Mexikaner, die sogenannten Criollos; jene mit gemischter ethnischer Herkunft, genannt Mestizos und die Indios, die 80\% der Bevölkerung ausmachten und von denen mehr als eine Million nicht einmal Spanisch sprachen, sondern in 42 verschiedenen Dialekten durcheinander redeten. Die imaginierte Nation, wie sie in der „Me- 
xicanidad“ Ausdruck fand, sollte Ängste vor kultureller, sozialer und ökonomischer und politischer Desintegration bannen.

Die Inventarisierung des „mexicano“ bildete den Kern dieser Ordnungsstrategie. Sie erfolgte - in Analogie zu modernen nationalistischen Strategien in Europa und in anderen lateinamerikanischen Ländern - durch die Festlegung von Typen und Mustern volkstümlicher Bilder, Traditionen und Werte des jeweiligen kulturellen Raums. Diese Homogenisierung kultureller Ausdrucksformen unter dem Signum des „Populären“ wurde dann zum „Volkscharakter“ stilisiert. Dafür gründete Vasconcelos ein Ministerium für öffentliche Bildung und Erziehung, führte Alphabetisierungskampagnen durch und versammelte Künstler und Literaten, die sowohl in den prähispanischen Traditionen Mexikos als auch in der Volkskunst den indigenen Ausdruck der „Mexicanidad“ suchten. Unter Vasconcelos entstand in den 1920er Jahren die Fiktion einer nationalen Geschichte in Mexiko, die nur ein einziges Subjekt kannte: „lo mexicano“. Von murales (Wandmalerei) über Literatur zu Körperkultur, Film und Musik fächerte sich die Produktion von Mythen, Symbolen und Bewegungsstilen auf, die „lo mexicano“ zugleich schufen und inventarisierten.

Diese Suche nach dem Volk, nach der ,authentischen“ nationalen Tradition und ihrem Symbolrepertoire erforderte die Zusammenarbeit mit den Künstlern. José Vasconcelos finanzierte die Rückkehr Diego Riveras aus Paris und bezahlte dessen Reisen in das Landesinnere gleich mit, wo Rivera jene Formen und Bildmotive entdeckte, die er in seinen „murales“ zum Ausdruck mexikanischer Volkskunst stilisierte. Auch die internationale Gruppe von Künstlern und Intellektuellen, die nach der Revolution Mexiko als Experimentierfeld für eine neue Kunst entdeckt hatten, erhielt staatliche Unterstützung für ihre Projekte. Die Konstruktion der kulturellen Selbstbeschreibung der Nation war letztlich das Ergebnis einer internationalen Zusammenarbeit, finanziert von den postrevolutionären Eliten. Die Amerikanerin Frances Toors brachte mit tatkräftiger Hilfe der Regierung Vasconcelos die Zeitschrift „Mexican Folkways“ heraus, die sich die Aufarbeitung volkstümlicher Kunst zur Aufgabe machte. Es war Diego Rivera, der den russischen Regisseur Sergej Eisenstein dazu motivierte, einen Film über Mexiko zu machen. Eisenstein und seine Crew (Grigori Alexandrov und Kameramann Eduard Tissé) hatten vorher in Westeuropa und den USA die neuesten filmischen Techniken erlernt. Sie reisten durch Mexiko und filmten Land und Leute, v.a. Bauern und Dorfbewohner. Eisenstein traf Künstler und Schriftsteller (u.a. Adolfo Best Maugard) und studierte Mexikos Geschichte und Bräuche.

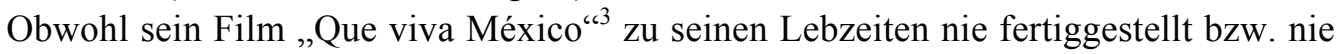
in einer von ihm akzeptierten Form veröffentlicht wurde (es existierten lediglich die von Eisenstein gemachten Auszüge „Thunder over Mexico“), hatte der unvollendete Film großen Einfluss auf mexikanische Filmemacher. Insbesondere auf der Grundlage seiner Folklorisierung des mexikanischen Raums etablierten nachfolgende mexikanische Regisseure einen nationalen Filmstil: Eisensteins Bilder von Landschaften und IndioGesichtern wurden zum Vorbild für unzählige Filme in den nächsten 20 Jahren. Das Kino erhielt eine besondere Bedeutung für die Erfindung nationaler Stereotype und einer nationalen Bewegungskultur. Vor allem die „Ranchera“, d.h. musikalische Melodramen, die auf folkloristischen Themen beruhten, waren für die mexikanische Identität das, was der Western für das nordamerikanische Selbstverständnis war.

3 Es existierte das Feature „Thunder over Mexico“ und Marie Seton, Eisensteins Biographin, nutzte Material aus diesem Film für „Time in the sun“(vgl. Mora 1982, 37). 
Politische Inszenierungen sollten dieses Ordnungsmodell zusätzlich im Tiefenraum der Gesellschaft verankern. So fand 1924 am Cinco de Mayo - dem Gedenktag der mexikanischen Revolution - ein nationales Massenspektakel im eigens dafür von der Regierung gebauten Nationalstadion statt. Dazu gehörten Massentänze im „mexikanischen Rhythmus“.

Die Erfindung einer mexikanischen Folklore zielte auf die Vereinheitlichung der Idiosynkrasien eines Landes, das über die Konstruktion von Regionen deren Homogenisierung in spezifische (stereotype) Ausdrucksformen - von Tänzen, über Handwerk, Architektur, Malerei bis hin zu Sitten und Gebräuchen - in Analogie zu europäischen Strategien der Nationenbildung einen kulturellen Kern von „Mexicanidad“ gegen innere und äußere Bedrohungen festschreiben wollte (vgl. Montfort 2000). Doch damit nicht genug: Auch die Chicanos in den ursprünglich mexikanischen und nun US-amerikanischen Territorien durften - finanziert durch die mexikanischen Konsulate in den USA - am Kulturexport der „Mexicanidad“ teilhaben. Diego Rivera und andere Künstler reisten in diese Gebiete und stellen ihre Arbeiten aus, die spanischsprachigen Kinos in den Barrios zeigten die mexikanischen Filme.

In dieses Projekt einer Nationalisierung der Kultur war von Anfang an ein unlösbarer Konflikt eingeschrieben: Die Migration zwischen Mexiko und den USA brachte beständig transnationale Formationen innerhalb des Nationalstaats hervor. Die Border-Crosser begleiteten die mexikanische Politik seit dem späten 19. Jahrhundert. Sie wurden von den Vertretern der „Mexicanidad“ voller Argwohn betrachtet, denn brachten die Migranten nicht kulturelle Werte und Verhaltensstile der feindlichen Nation ins Land, die die nationale Ordnung zu zersetzen drohten? Mit der Migration zwischen Mexiko und den USA entwickelte sich eine Border-Culture, die sich mit den Erfahrungen der Wanderer zwischen den Welten auseinandersetzte. Jenseits der offiziellen Kulturpolitik der „Mexicanidad" entstanden dabei neue kulturelle Ausdrucksformen, die von der Regierung nicht gern gesehen wurden, weil sie sich im nationalen Modell nicht verorten ließen. Es kam zu immer neuen Abspaltungen. Die Border-Crosser und Migranten galten als „Verräter“ und Abtrünnige der nationalen mexikanischen Familie.

Allerdings gab es bereits Gruppen, die aus mexikanischer, aus nordamerikanischer wie aus Chicano-Perspektive als verdächtig galten: die „Pachucos“. Diese waren zumeist Mexikaner der zweiten Generation, Kinder der Arbeitsmigranten. Seit den 1920er und 30er Jahren formierte sich diese urbane Subkultur in den USA, die ihre Gruppenzugehörigkeit nicht nur in der Sprache - sie sprachen „caló“, einen Slang, der aus einer Mischung aus Englisch und Spanisch mit Wortneuschöpfungen bestand - sondern auch im Bewegungsstil, in den Frisuren und der Kleidung manifestierten. Die „zoot suits“ waren mehrere Nummern zu große Anzüge mit wattierten Schultern, dazu gehörten manchmal eine am Gürtel festgemachte Kette und lange, nach hinten gekämmte Haare. Pachucos erfanden eine symbolische Verkleidung, die den zoot-suiter als jemanden identifizierte, der weder der mexikanischen noch der nordamerikanischen Kultur angehörte. Daher waren sie auch den Chicanos, die gerade ihre Gemeinschaft wieder nach dem Modell einer eigenständigen „Nation“ imaginiert hatten, ${ }^{4}$ ein Dorn im Auge.

4 Dies gilt bis in die 1960er und 1970er Jahre. „Nationalism as the key of organization transcends all religious, political, class, and economic factions or boundaries. Nationalism is the common denominator that 


\section{Border-Crossing: Das Ordnungsmodell der Postnation}

Seit Mitte der 1980er Jahre entdeckten Künstler und Intellektuelle die Grenze als Experimentierfeld der Postnation, das globale Gültigkeit beanspruchte.

Die Border-Culture hat ihre Vorläufer in den beschriebenen transnationalen Phänomenen der 1930er und 1940er Jahre. An der Grenze zwischen Mexiko und den USA wurde die Border-Culture zu einem Paradigma für transkulturelle Übergänge und damit für eine neue Politik des Raums, die überkommene Verbindungen von Kultur und Raum wie auch von Identität und geographischer Herkunft aufheben sollte. Poststrukturalistische Theorie aus Frankreich in Verbindung mit Theorieangeboten der Postcolonial Studies lieferten über nordamerikanische Universitäten den Rahmen für neue Modelle postnationaler Identitäten, die auf die Anforderungen und Schwierigkeiten eines Lebens „Zwischen“ den Kulturen reagierten, wie sie das Leben an der Grenze oder der Chicanos oder der ,undokumentierten“" Mexikaner (= Migranten ohne Ausweispapiere) in den Metropolen der nordamerikanischen Großstädte charakterisieren. Migranten und Border-Crosser erklärte man zu Wegbereitern für neue Konzepte von Identität. Bewegung bzw. Entortung galt als Grundlage dieser relationalen cross-cultural identities einer globalisierten Welt.

Eine Schlüsselfigur dieser Bewegung ist der in Los Angeles und Mexico-City lebende Mexikaner Guillermo Gómez-Peña. Der Schriftsteller und Performance-Künstler war einer der ersten, der in den 1980er Jahren den Begriff der Grenze zum Mittelpunkt eines neuen Kulturverständnisses machte. Gómez-Peña beschrieb die Erfahrung des BorderCrosser als eine Fusion und Verschiebung von Kulturen, eine ,juxtaposition of images, sounds, and texts without demanding reconciliation or rationalization“ (Gómez-Peña 1995, 209) innerhalb einer Raum-Zeit-Heterotopie, in der sich Archaisches, Modernes und Postmodernes überlappen. Diese Idiosynkrasien auf verschiedensten Ebenen wurden in der Performance-Kunst von Gómez-Peña systematisch ausgelotet. „Crossing the border - that in itself is involuntary postmodernism. You cross the border and in a matter of seconds you move from Catholicism to Protestantism, from the past to the future, from Spanish to English, from pre-Columbian to high tech, from hedonism to Puritanism. This experience of disjunction, this experience of rupture, is a quintessential contemporary experience. In our work, we ask, what happens when Mexican corrido (ballad) meets video? What happens, when a little indian altar meets audio art? What happens when Mexican carpa (popular traveling theater) meets multimedia.“ (Gómez-Peña 1995, 156)

Aus dem ständigen Überqueren der Grenze, so die frohe Botschaft, bringe der BorderCrosser eine Identität zwischen den Kulturen hervor. Auch die Chicanos galten unter diesem Aspekt als veraltet, denn für sie hatte sich das Konzept der "Grenze“ von einer Grenzlinie zu einem eigenständigen Grenzgebiet gewandelt, das als Zwischen-Raum selbst wieder wie eine Nation behandelt wurde. Eben diese „Nation“ aber sollte ausgeschlossen werden: Grenzgebiet - so hieß es nun - meine nicht mehr ein Diesseits oder Jenseits der Grenze, sondern erfasse den Raum, der nach mehreren Richtungen hin offen sei. ${ }^{5}$ Durch die Vermischung der Sprachen und kulturellen Deutungsmuster entstünden zusätzliche Register für die Artikulation von etwas Drittem. Die postmoderne Rhetorik für neue transnationale Identitätsmodelle zu politisieren, war das Anliegen dieser Rezep-

all members of La Raza can agree upon,“ heisst es im „Spiritual Plan of Atzlán“, dem Manifest der Chicano-Gemeinschaft von 1969 (zit. nach Barrera 1988, 38).

5 „Border“ entwickelt sich mittlerweile in den nordamerikanischen Cultural Studies zu einem epistemologischen Modell (vgl Saldívar 1997, 185/186). 
tion. Dazu gehörte insbesondere das Konzept der Übersetzung, wie es der französische Philosoph Jacques Derrida formulierte. Für die Border-Culture wurde daraus eine Praktik der Künstler bzw. Kulturkritiker (häufig in Personalunion wie im Fall von Gómez-Peña), die Theorien und Konzepte transformiert, indem diese in neue Kontexte transponiert und dort revidiert, adaptiert und neu artikuliert werden. Aber war das wirklich alles so neu?

Wie ihre Vorläufer erhielt die Border-Culture ihre Konturen überhaupt erst als Gegenentwurf zur „Mexicanidad“. Wiederum waren internationale Künstler und Intellektuelle am Werk, die nun dekonstruktivistische Diskurse aus Europa über die USA rezipierten. Die Funktion der US-Mexikanischen Grenze als Paradigma eines neuen Verständnisses von kultureller Identität und der Gleichsetzung von kultureller Identität und geographischem Raum problematisierten die Cultural Studies in den USA. Die Grenze wurde zu einem ,paradigm of crossings, intercultural exchanges, circulations, resistances, and negotiations as well as of militarized 'low-intensity conflict"“ (Saldívar 1997, IX). Dabei entstand ein Imaginäres der Grenze, das als „,border“ oder „,borderland“ insbesondere die Chicano-Studies in den USA faszinierte, die sich mit neuen Verbindungen zwischen Nation und Transnation auseinandersetzten. Traditionelle Raummetaphern wie z.B. Land, Zentrum und Peripherie wurden durch neue Bilder wie „borderland“, Grenzzone oder Kreislauf (circuit) ersetzt.

Die poststrukturalistische Vorliebe für Grenzüberschreitungen, für die Auflösung von Gattungsgrenzen und liminale oder „Zwischenraum“-Existenzen fand in der Erfahrung der Migranten und Border-Crosser eine Entsprechung. Allerdings war diese Alltagserfahrung - bevor die Border-Culture sie zum Paradigma postnationaler Lebensformen erhob eher eine anstrengende, schmerzhafte und zuweilen lebensgefährliche Existenzform.

Die Vorstellung homogener nationaler Kulturen und ihrer von einer Gemeinschaft getragenen Überlieferung war allerdings schon im frühen 20. Jahrhundert eine Fiktion. Auch die aktuelle kulturelle Landschaft nationaler Kulturen ist in einem Prozess grundlegender Neubestimmung und Neuverhandlung begriffen. Schon seit Mitte der 1970er Jahre erlebten die USA eine Krise des nationalen Selbstverständnisses, weil das alte Konzept kultureller Einheit auf der Basis des Schmelztiegels USA - des berühmten „Melting Pot“ - nicht mehr funktionierte. Es entwickelte sich ein neues Verständnis von Kultur, das die Pluralität und die Differenz zwischen Kulturen propagierte. Eine neue Identitätspolitik, die mit dem Konzept von „Multikulturalismus“ arbeitete, ersetzte den veralteten „Melting Pot" durch die Idee von einer multikulturellen Gesellschaft. Angesichts neuerer Erfahrungen mit Migration und Migrationskulturen erweisen sich aber auch diese Vorstellungen als problematisch. In kritischen und historischen Diskursen assoziierte man Konzepte wie „Hybriditität“ und „Borderlands“ mit Auflösung und Fragmentierung und sah in ihnen eine Herausforderung für Modelle, die Identität noch nach dem Schema des Zusammenfallens von Ort, Zuhause und Souveränität sowie nach nationalen und sogar wissenschaftsdisziplinären Gesichtspunkten verstanden.

Durch die Migrationserfahrung - so die theoretische Überhöhung ihrer Existenz - bilden die Border-Crosser spezifische Kompetenzen heraus, beispielsweise eine besondere Sensibilität für die Vermittlung zwischen traditionellen Lebensformen, Symbolen, Mythen und hochtechnisierter Audiovision. Über die Vermischung der Sprachen und kulturellen Deutungsmuster würden sich für die Artikulation von etwas Neuem zusätzliche Register entwickeln. Natürlich erhob die „Border-Theorie“ den Anspruch, keine abgehobene Theorie zu sein, sondern die Alltagserfahrungen der lokalen und subalternen Gemeinschaften der Grenze theoretisch zu reflektieren. Darauf bestand 1996 zumindest Guillermo Gómez-Peña: „Here/there we are all potential border-crossers and cultural exiles. We have all been uprooted to different degrees, and for different reasons, but not 
everyone is aware of it. Here/there, homelessness, border culture, and deterritorialization are the dominant experiences, not just fancy academic theories." (Gómez-Peña zit. nach Bejarano 2005, 25)

Fühlten sich die internationalen Künstler und Intellektuellen, die in den 1920er und 30er Jahren das Projekt der „Mexicanidad“ vorantrieben, als Vorreiter einer neuen (mit internationalen Referenzen versehenen) Nationalkultur, so war das Selbstverständnis der Künstler und Intellektuellen der Border-Culture das eines ,kulturellen Übersetzers“, der ähnlich wie Migranten und Border-Crosser - eine besondere Fähigkeit der transkulturellen Kommunikation ausbildet, die als symbolisches Kapital für eine neue postnationale Identitätspolitik nutzbar gemacht werden sollte. Die Künstler der Border-Culture fanden seit den 1980er Jahren ein immer breiteres Publikum - nicht nur in Lateinamerika oder in den USA, sondern auch in Europa. Denn auch hier wurde ihre Arbeit als Paradigma für aktuelle Prozesse der Globalisierung interessant: Gómez-Peñas Auftritte in London, Barcelona oder Berlin sind nur ein Beispiel dafür.

\section{Neue Heilige und die Ausweitung der Nation}

Die imaginierte Gemeinschaft der „Mexicanidad“ und ihr Symbolrepertoire hatte starke sakrale Dimensionen, denn die damit verbundene Naturalisierung des Nationalen bot Deutungsmuster für Lebensformen, deren sinn- und orientierungsstiftende Wirkung mit religiösen Bildwelten vergleichbar ist. Darin lag möglicherweise einer der Gründe für ihren Erfolg - nicht nur in Mexiko. Denn auch die Chicanos orientierten sich an dem nationalen Modell, das seit Ende der 1960er Jahre im Zuge der in den USA entstandenen ethnischen Identitätspolitik als „Chicano-Nation“ umgeschrieben wurde. Den Bruch mit diesem Konzept vollzog erst die Border-Culture der 1980er Jahre. Sie propagierte postmoderne Lebensentwürfe, aber keine Lebensformen für breite Bevölkerungsschichten.

Die Border-Culture führte nicht zur Abschaffung der Nation, sondern zu deren „Modernisierung“ im Sinne einer Ausweitung ihrer Konzepte von Citizenship. Dazu gehört nicht nur die Möglichkeit einer doppelten Staatsbürgerschaft (vgl. Castañeda 2006), sondern auch, dass die Nation nicht mehr als essentialistische Kategorie, sondern als Konstrukt verstanden wird, das seine Konstruiertheit vorführt. Doch auch diese Variante ist schon wieder veraltet. Es entstehen spontan neue Kulte aus den kollektiven Hoffnungen und Ängsten, die das Leben im Grenzgebiet mit sich bringt. Sie bedienen sich religiöser Symboliken, stehen aber den etablierten Kirchen fern. Die Drogendealer (narcotraficantes) haben ihren Schutzpatron („Jesús Malverde“), aber auch die Heilige „Santa Muerte“ zu denen sie beten, denen sie Opfergaben bringen, für die sie Votivbilder (ex-votos) aufstellen und Straßen-Altäre errichten. Sie schmücken mit ihren Bildern den heimischen Hausaltar, so wie es die Border-Crosser und Migranten auch tun. Letztere fanden in der Figur des „Juan Soldado“ ihren Patron; er war ein in den 1930er Jahren in Tijuana schuldlos für ein Verbrechen verurteilter und erschossener Soldat. Vor einem geplanten Grenzübergang bitten sie ihren Heiligen um Schutz, zünden Kerzen an oder schmücken nach dem Gelingen seinen Altar mit Blumen, Gedenktafeln und Votivbildern als Dank dafür, dass sie nicht von der „Migra“, der Grenzpolizei, erwischt wurden oder dafür, dass sie mit Erfolg an offizielle Dokumente gelangt sind. Diese Kulte funktionieren wie neue Ordnungsmodelle von unten. Es handelt sich dabei um eine indirekte Stabilisierung, die davon zeugt, dass in einer Situation globaler Entgrenzung lokale und nationale Zugehörigkeitsgefühle nicht verschwinden, sondern sakrale Verhaltensdispositionen, wie sie 
auch die nationale Gemeinschaft produzierte, immer noch oder schon wieder Stabilität vermitteln.

Trotz aller postnationaler Rhetorik der Border-Culture wie auch aller Warnungen konservativer Nationalisten vor der ,inneren Aufweichung“ der Nation infolge von Migration und Mischkulturen hat sich die Nation als äußerst flexibler Rahmen erwiesen, der durchaus in der Lage ist, neue Ansprüche zu integrieren. Aktuelle Globalisierungsprozesse stellen die Frage nach der Perspektive von nationalen Identitätskonstrukten auf neue Weise. Die Tendenz zur Ablösung von Kulturgemeinschaften von geographischen Orten durch Globalisierung und neue Medientechnologien (insbesondere dem Internet) wirft die Frage auf, ob wir nicht alle zu „Nationen im Dritten Raum“ werden, die das aus der Moderne überkommene Konzept von Nation unterhöhlen. Welche Folgen hat diese Entwicklung für Identifikationsprozesse, für die Rituale nationaler bzw. Kultur- Gemeinschaften, für ihre historische Identitätsbildung? Das Ende der Border-Culture als postnationales Modell wurde nicht erst mit der von Samuel P. Huntington initiierten Debatte über die Rolle der Mexikaner in den USA oder der aktuellen Grenzverschärfung der USA gegenüber Mexiko offensichtlich. Während Huntington die mexikanischen Immigranten innerhalb der USA als „innere“ Aufweichung der Grenze zwischen beiden Nationalstaaten und Bedrohung nordamerikanischer kultureller Identität ansieht, ${ }^{6}$ stellt sich am Ende der postnationalen Euphorie die Frage nach dem „Trans“ in der Nation auf neue Weise. Transnationale Formationen und nationale Kultur waren nie ein Widerspruch, sondern führen wie das Beispiel der „Mexicanidad“ und das Scheitern der Border-Culture zeigt - zu einer Reorganisation nationaler Kultur. Die Rekonstruktion der „verborgenen“ Geschichte der Heterogenität im homogenen Nationalstaat muss daher nicht in Forderungen nach Denationalisierung einmünden. Die kulturellen Praktiken der Migranten zwischen Mexiko und den USA stellen multilokale Gemeinschaften her. Diese neuen Räume nationaler Gemeinschaft spielen eine entscheidende Rolle um die Frage zu beantworten, was Staatsbürgerschaft in der Praxis bedeutet. Sie tragen dazu bei, die überkommenen Modelle von Nationalstaat und Staatsbürgerschaft zu flexibilisieren.

Es sieht so aus, als entstünden neue kulturelle Nationen in verschiedenen Milieus, die nicht unbedingt stabil sein müssen, und die daher mit den alten Staatsnationen nicht mehr viel gemeinsam haben. Eben deshalb sind sie aber auch nicht die befürchtete Bedrohung, wie von den nationalistischen Theoretikern angenommen. Umgekehrt sind die herkömmlichen Nationen flexibler, als die Border-Theoretiker angenommen hatten, denn sie lernen, mit diesen subkutanen „Nationen“ zu leben; es ist sozusagen ein nationales Imaginäres auf zwei Ebenen entstanden, das sich teilweise überschneidet, sich aber nicht wechselseitig ausschließt. Ein vereinzelter „Nomade“ zu sein, mag vielleicht für einen internationalen Intellektuellen zum konstitutiven Lebensgefühl werden; als normative

6 "Mexican immigration is leading towards the demographic reconquista of areas Americans took from Mexico by force in the 1830s and 1840, Mexicanizing them in a manner comparable to, although different from, the Cubanization that has occurred in southern Florida. It is also blurring the border between Mexico and America, introducing a very different culture, while promoting the emergence, in some areas, of a blended society and culture, half-American and half-Mexican.[...] Mexican immigration has these effects because of the characteristics that differentiate it from past and present immigration from other countries and because of the extent to which Mexican immigrants and their progeny have not assimilated into American society as other immigrants did in the past and as many other immigrants are doing now." (Huntington 2005, 224/225) [Hervorhebung im Text] 
Vorschrift für eine ganze Bevölkerung, auch wenn sie in einer expliziten Grenzregion lebt, taugt es kaum zum Vorbild. Die Nation fasziniert weiterhin und ist offenbar auch in Zeiten der Globalisierung nicht abzuschaffen. Die Frage ist also nicht, $o b$ nationale Kulturen weiter existieren, sondern es geht um die Form, in der sie leben. Die mexikanische Nation war in ihren Bestandteilen immer schon transnational. Und diese Transnationalität ließ sich nur bedingt zu einem einheitlichen Gebilde verschmelzen. Unter der Hülle der Nation wucherte das transnational-revolutionäre Chaos weiter. Bis in die Gegenwart hinein hat es verschiedene kulturelle Entwürfe für diese Grenzregion gegeben, die sich stellenweise überlagern, die eine Zeit lang als eigenständige Border-Culture gefeiert wurden, die sich aber letztlich dem Sog einer - wenn auch transnationalen - Nation nicht entziehen konnten. Es lässt sich durchaus die These vertreten, dass diese Modernisierungsschübe, die ihrer Intention nach das Ende des Nationen-Konzepts im Blick hatten, letztlich zur Modernisierung und zum Überleben eines bereits verabschiedeten Modells beigetragen haben.

\section{Bibliographie}

Anderson, B. (2006 [1982]) Imagined communities: On the origin and spread of nationalism. London.

Baxmann I. (2007) Mayas, Pochos und Chicanos. Die transnationale Nation. München.

Barrera, M. (1988) Beyond Atzlán. Ethnic Autonomy in Comparative Perspective. New York.

Bejarano, C. L. (2005) ?Qué onda? Urban Youth Culture and Border Identity. Tucson/Arizona.

Castañeda, A. (2006) The Politics of citizenship of Mexican migrants, New York.

Gómez-Peña, G. (1995) On the Threshold of a New Decade.The End-of-the-CenturySociety. In: Gere, D. (ed.) Looking Out. Perspectives on Dance and Criticism in a Multicultural World. New York.

Hodges, D. C./Gandy R. (2002) Mexico. The end of the Revolution. Westport, Connecticut, London.

Huntington, S. P. (2005) Who are we? America's Great Debate. London.

Montfort, R. P. (2000) Avatares del nacionalismo cultural. Cinco ensayos. México D.F.

Mora, C. J. (1982) Mexican Cinema. Reflections of a Society 1896-1980. Berkely/Los Angeles/London.

Saldívar, J. D. (1997) Border Matters. Remapping American Cultural Studies. Los Angeles.

Inge Baxmann, Professorin am Institut für Theaterwissenschaft der Universität Leipzig; Studium der Romanistik und Komparatistik in Bochum und Paris; wissenschaftliche Assistentin am Institut für Französische Literaturwissenschaft der TU Berlin; Postdoktorandin am Graduiertenkolleg „Kommunikationsformen als Lebensformen“ an der Universität GHS Siegen; wissenschaftliche Mitarbeiterin der Projektgruppe „Kultursemiotik“ am Zentrum für Literaturwissenschaft Berlin; Habilitation im Fach Kulturwissenschaften an der Humboldt-Universität Berlin.

Forschungsschwerpunkte: Symbolisierung und Inszenierung der Nation zwischen Französischer Revolution und Gegenwart; Körperwissen und die Umstrukturierung der Wissenskulturen in der Moderne

E-mail: inge.baxmann@snafu.de 